\title{
High Performance Virtual Backup and Archive System*
}

\author{
Dan Feng, Lingfang Zeng, Fang Wang, and Peng Xia \\ Key Laboratory of Data Storage System, Ministry of Education \\ School of Computer, Huazhong University of Science and Technology, Wuhan, China \\ dfeng@hust.edu.cn, zenglingfang@tom.com
}

\begin{abstract}
Built on a sequential write/read device, a tape library is seldom considered as a viable place for fast backup/restore data. With the help of the virtualization technology, in this paper we propose a virtual backup and archive system, called VBAS. The purpose of VBAS is to maintain a consistent view of mass storage so that the user can effectively manage it. And VBAS allows users to create files and directories as well as delete, open, close, read, write and/or extend the files on the device(s). VBAS maintains security on the files and provides the management for fragmentation. Moreover, VBAS can support large-scale file systems. Users have two ways to access VBAS: using general backup application, and through the APIs provided by VBAS. Based on VTL, RAID-DP, and iSCSI, VBAS not only has the disk-file-system-like functions, but also retains the characteristics of tape library storage, thus achieving a good tradeoff between cost and performance. The prototype system performance is presented and improvements are analyzed to achieve higher write/read performance.
\end{abstract}

\section{Introduction}

Tape is by far the most popular media for the near-line or offline (e.g. archiving data) storage. As the amount of stored data in many data-intensive applications, such as high energy physics research, weather prediction, spatial data and seismic data analysis, increases dramatically, tape libraries can play an important role in maintaining and backing up critical data. The disadvantage of tape drives is that they are sequential-access devices. This makes them much too slow for general-purpose storage operations.

The primary driving force behind users' adoption of archiving has traditionally been to reduce total cost of ownership (TCO). Though cost is often thought to be the most important factor, it ranks lower than data retrieval performance and scalability in the selection criteria for an archiving solution. Users want to achieve file retrieval in minutes or even seconds.

* This paper is supported at Huazhong University of Science and Technology by the National Basic Research Program of China (973 Program) under Grant No. 2004CB318201, National Science Foundation of China No.60273074, No.60303032, Huo Yingdong Education Foundation No.91068. 
Nowadays, many file systems based on disk and tape storages have been presented. AMASS [1] for UNIX, designed for direct-attached and network-attached storage environments, is a cost-effective solution for enterprises that have more data than their disk capacity can support. AMASS transforms libraries into truly online direct-access mass storage. AMASS presents an automated optical, tape, or DVD library as one device and one mount point (showed in Figure 1.), through a standard UNIX file system interface consisting of directories and files. Users or applications can save or access files residing on libraries in the same way they would with magnetic disk. AMASS provides transparent access to data on disk and tape library storage.

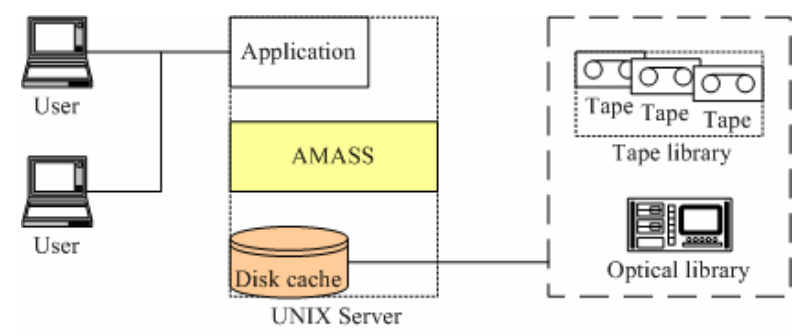

Fig. 1. AMASS provides transparent access to data on disk and library storage

However, disks in AMASS are used based on the general-purpose file system, and thus they have to confront the same set of problems inherited from general-purpose file systems.

SEPATON's Disk Dynamic File System allows large I/O streams to execute efficiently and has the built-in infrastructure to dynamically balance performance across all available disks in their VTL appliance. The Disk Dynamic File System has the important side effects of not only sustaining maximum throughputs, but also dynamically load balancing I/O streams without any requirement for performance "tuning" [2]. But, The Disk Dynamic File System is still a disk-related file system and it is not feasible for the management of tape library storage system.

Our virtual backup and archive system (VBAS) effectively integrates the virtual tape library (VTL) [3], the VTS technology [10], the RAID-DP technology [4], and the iSCSI [5] technology to provide transparent tape file access for users while retaining other functions of tape libraries. Our study of VBAS shows the main advantages of VBAS over the conventional tape libraries and conventional (disk-based) file systems as follows: (1) High backup and restore performance with disk-file-system-like functions. (2) Low cost compared with simple disk-based system. (3) Some finer functions integrated both RAID and the tape library.

The main contributions of this paper are: (1) Overcomes general file systems more prone to being infested with viruses and having an inherent problem of fragmentation. (2) Provides a virtual backup and archive system based on RAID. (3) Implements the prototype system via virtual tape library technology and presents and discusses the experiment results.

The rest of the paper is organized as follows. The design and implementation of VBAS is presented in the section that follows. Test results and performance analysis 
are discussed in Section 3. Finally, we give the conclusions and outline some remaining problems in Section 4.

\section{Design and Implementation of VBAS}

VBAS enables data management without disrupting end-user or application accesses. Done correctly, the VBAS can reduce storage management overhead, simplify end-user accesses, and enable additional storage management functionalities. The VBAS solutions transform a mass storage system from a "two-size-fits-all" (high-performance disk and low-cost tape) world into a sophisticated mix of discrete storage elements with a wide variety of performance, cost and capacity attributes. The alphabet soup of technologies (VTL, iSCSI, SATA, etc.) presents an excellent opportunity for the storage systems applications to locate the right data on the right device at the right time.

\subsection{Hardware Architecture of a VBAS-Based Testbed}

Figure 2 shows the hardware architecture of our mass storage system testbed based on VBAS. The target device comprises a RAID and a tape library connected by a SCSI channel, also called a hybrid device. The console and application servers (Web server, E-mail server etc.) and the target device are interconnected by a TCP/IP network. The console, web server, e-mail server and backup server form an initiator and they access data in the hybrid device through the iSCSI protocol.

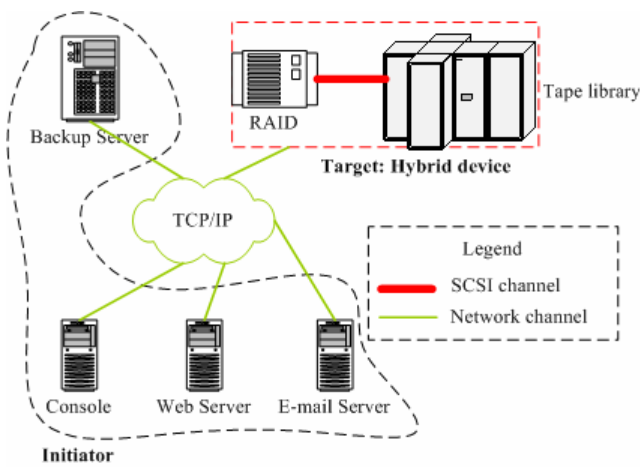

Fig. 2. Hardware architecture of the mass storage system testbed based on VBAS

\subsection{The VBAS-Based Software Deployment}

iSCSI builds on the two most widely used protocols from the storage and the networking worlds. From the storage side, iSCSI uses the SCSI command set, the core storage commands used throughout all storage configurations. On the networking side, iSCSI uses IP and Ethernet that form are the basis for most enterprise networks and used in metropolitan and wide area networks. 
With the help of iSCSI, shown in Figure 2, almost all of backup applications, such as tar [6], taper [7] and bacula [8], can access the target (hybrid device) via VBAS. It is possible that, for a backup application, the daemon servers may be deployed in the backup server while its proxies may be installed in the web server or E-mail server.

Figure 3 shows the functional and logical relationship among the function modules in the initiator and the target. The target device refers to an entity that presents itself as a SCSI direct access disk and tape sequential access while running within the Linux kernel space. In the user space implementation, the entity providing the SCSI functionality and the entity responsible for transmitting SCSI over a given protocol form one logical piece of code. However, in the kernel space implementation, the entity responsible for handling SCSI commands has an existence independent of the low-level front-end target driver that is responsible for the transmission of SCSI in a device-specific manner. Thus, in terms of visualization, it may be better to think of the kernel space modules as consisting of two distinct entities - the generic SCSI target mid-level and the low-level front-end protocol-specific target driver. The VBAS module is implemented in the initiator. It can work with the traditional disk-related file system and this solution is transparent for user space application. Also, users can custom their applications based on the APIs provided by VBAS.

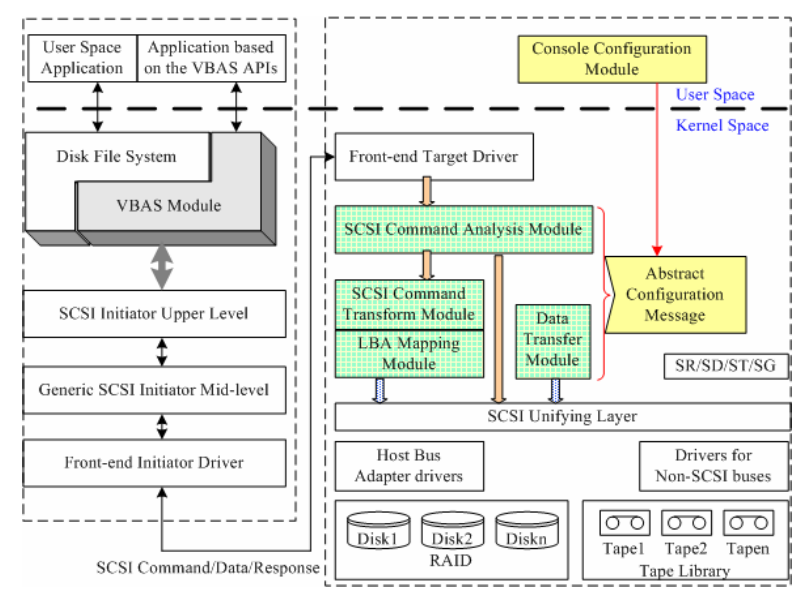

Fig. 3. The functional and logical relationship among the function modules of the prototype system

In the target, our function modules are implemented in SCST (The SCSI target mid-level subsystem for Linux) [9], which is a generic SCSI target middle level for Linux. It is designed to provide unified, consistent interface between SCSI target drivers and Linux kernel and simplify target drivers development as much as possible. Although data distribution policy is different comparing with [10] [11], in substance, their implementation technologies are analogical. And they all have to record all the logical objects [12] on the RAID. In Figure 3, the SCSI command analysis module receives SCSI sequential commands from the backup application, and determines whether the commands should be executed on the RAID or on the tape library. Then it delivers them to the proper module (or media). The SCSI command transform module 
is responsible for transforming SCSI sequential commands into SCSI block commands. The LBA (logical block address) mapping module maintains the block mapping information, which associates the logical unit of an object with its logical block address in the RAID. The data transfer module performs data transfer between RAID and tape library according to some information lifecycle management policy.

In addition, VBAS provides a client application that is implemented using the API of VBAS. The client application may be deployed in the console (Figure 2.) and perform remote file management operations. Users also can implement their own remote file management application by the API of VBAS.

\subsection{The VBAS Data Structures}

Traditionally, a file system represents the logical structures and software routines used to control access to the storage on a hard disk system. However, VBAS, a tape-based file system that hides the details about tapes and, provides an API for applications, is designed and implemented to provide the "access by name" functions, including tape file creation, tape file read, tape update, tape file deletion, tape file copy, tape file renaming and tape defragmentation.

1. VBAS data structures and functions

The file allocation table (FAT) of every library slot and every tape are defined. The FILE_NODE constructs the file name information and the FILE_RECORD records some information of file in true tapes. The virtual tape list and logical object list are defined. For the type element, its value may be one of the defined constants LOGICAL_BLOCK, FILE_MARK, SET_MARK, BEGIN_NODE or END_NODE.

Some main functions (in target software) dispose the transform from SCSI stream command to SCSI block command, such as INQUIRY (0x12), REWIND (0x01), READ (0x8), WRITE (0xA), MODE_SENSE (0x1A), WRITE_FILEMARKS (0x10), SPACE (0x11) etc.. Specially, for the RAID, the command type of write and read is 10 , so the VBAS has two transform functions (transform_write_6to_10 and transform_read_6to_10).

2. The system/configure files in VBAS

At the same time, VBAS provides some system files and configure files which facilitate the configuration about VBAS. The system files record some file metadata information in VBAS, and the configure files provides the configure information of VBAS. For instance, the TapeLibrarySlotInfo.txt records the tape library slot information (defined by SLOT_FAT) both in the VTL and the true tape library. The TapeVBASFAT.txt stores the information of tape file allocation table (defined by TAPE_FAT) in VBAS. The FileName.rec gives file name information (defined by FILE_RECORD), and the configure information for the VBAS is set or got by the administrator.

3. File operation algorithms in VBAS

This subsection shows those file operation algorithms, such as create, dir, read, update, copy, erase, rename and defragment etc. For each file operation algorithm, it begins at the initialization file system function - InitFileSystem(), and ends at the close file system function - CloseFileSystem(). Because those algorithms aim at the write or read files in a tape, they are all applicable to both the true tape library and the VTL. Also, as mentioned above, in the initiator, users can also use the APIs provided by the 
VBAS to implement tape file management. Key file operation algorithms for VBAS are as follows (update, copy, erase, rename and defragment are omitted):

\section{Create \{}

1: Open the file TapeLibrarySlotInfo.txt in VBAS, create read/write buffer, initiate the process of read/write and open the device file (e.g. st 0 or nst0) of VTL.

2: Judge if the online VTL tape runs out of space or not. If the tape runs out of space, exchange a new VTL tape.

3: Deal with the name confliction according to the SLOT_FAT and open the file of tape file allocation: TapeVBASFAT.txt, and form the current TAPE_FAT.

4: Write data to the buffer.

5: The write process waits for that the buffer becomes full, and writes data to the VTL tape.

6: Write the remainder data in the buffer and close the FileName.rec, TapeVBASFAT.txt and TapeLibrarySlotInfo.txt.

7: Free the read/write buffer. And stop read/write process and close the device file of VTL.

\}

\section{Dir \{}

1: Open the file TapeLibrarySlotInfo.txt in VBAS, create read/write buffer, initiate the process of read/write and open the device file of VTL.

2: Read the every item in the TAPE_FAT of VBAS and list them one by one.

3: Close the VBAS and close the device file of VTL.

\}

\section{Read \{}

1: Open the file TapeLibrarySlotInfo.txt in VBAS, create read/write buffer, initiate the process of read/write and open the device file of VTL.

2: Judge if the required file is in the online VTL tape or not. If not, exchange a new VTL tape, and open the files: TapeVBASFAT.txt and FileName.rec.

3: The read process reads the file to the buffer according to the first address of the TAPE_FAT and the FILE_RECORD.

4: The application reads data from the file buffer by the read function of VBAS.

5: Close the FileName.rec, TapeVBASFAT.txt and TapeLibrarySlotInfo.txt.

6: Free the read/write buffer and stop read/write process and close the device file of VTL, close VBAS.

\}

\section{Test Results and Performance Analysis}

To test the write/read performance of VBAS, Windows 2000 and Redhat Linux were used in front-end servers. Under Windows 2000, we adopted Auto Backup. Under the Redhat Linux system (Linux kernel 2.4.20-8), we adopted tar and Taper [7] as the backup software. These three kinds of backup software performed well with VBAS. The results indicate that VBAS has compatibility with multiple operating systems and backup software.

We tested the performance of VBAS by using taper under Redhat Linux (also Linux kernel 2.4.20-8). The tape drive we adopted was HP MSL5030, and the tape media was 
hp ultrium 200GB data cartridge (C7971A). We adopted the SEGATE ST3404LC SCSI disk to simulate a VTL tape.

Our main concern is the backup time (write performance) and the restore time (read performance) for different primary backup devices. Also, the write/read performance both in different Ethernet and in the local node was tested in our experiment. Table 1 and 2 show the results. There are four group data, and each group recorded those test results about the write time and the read time. Moreover, we adopted different number of file, and the total size of file(s) was also ranked from 50Mbyte to 1 Gbyte.

Table 1. Throughput in different Ethernet

\begin{tabular}{|c|c|c|c|c|c|}
\hline \multirow[b]{3}{*}{$\begin{array}{c}\text { Number } \\
\text { of file }\end{array}$} & \multirow{3}{*}{$\begin{array}{l}\text { Total } \\
\text { size } \\
\text { of file } \\
\text { (Mbyte) }\end{array}$} & \multicolumn{4}{|c|}{ The VTL tape in VBAS } \\
\hline & & \multicolumn{2}{|c|}{ 1000M Ethernet } & \multicolumn{2}{|c|}{ 100M Ethernet } \\
\hline & & $\begin{array}{c}\text { Write } \\
\text { Throughput } \\
\text { (Mbyte/Min) }\end{array}$ & $\begin{array}{c}\text { Read } \\
\text { Throughput } \\
\text { (Mbyte/Min) }\end{array}$ & $\begin{array}{c}\text { Write } \\
\text { Throughput } \\
\text { (Mbyte/Min) }\end{array}$ & $\begin{array}{c}\text { Read } \\
\text { Throughput } \\
\text { (Mbyte/Min) }\end{array}$ \\
\hline 1 & 50.0 & 1509.7 & 1000.0 & 603.8 & 750.0 \\
\hline 1 & 200.0 & 1182.0 & 705.9 & 1006.4 & 571.4 \\
\hline 1 & 1000.0 & 1115.1 & 714.3 & 1090.0 & 566.0 \\
\hline 381 & 85.0 & 850.6 & 1020 & 850.6 & 850.6 \\
\hline 3386 & 455.1 & 684.7 & 941.6 & 681.2 & 910.2 \\
\hline 22216 & 1000.0 & 503.9 & 759.5 & 503.9 & 659.3 \\
\hline
\end{tabular}

Table 2. The throughput comparison of the local node of VTL and the physical tape

\begin{tabular}{|c|c|c|c|c|c|}
\hline \multirow[b]{2}{*}{$\begin{array}{c}\text { Number } \\
\text { of file }\end{array}$} & \multirow{2}{*}{$\begin{array}{c}\text { Total } \\
\text { size } \\
\text { of file } \\
\text { (Mbyte) }\end{array}$} & \multicolumn{2}{|c|}{ The VTL in Local Node } & \multicolumn{2}{|c|}{ The Physical Tape } \\
\hline & & $\begin{array}{c}\text { Write } \\
\text { Throughput } \\
\text { (Mbyte/Min) }\end{array}$ & $\begin{array}{c}\text { Read } \\
\text { Throughput } \\
\text { (Mbyte/Min) }\end{array}$ & $\begin{array}{c}\text { Write } \\
\text { Throughput } \\
\text { (Mbyte/Min) }\end{array}$ & $\begin{array}{c}\text { Read } \\
\text { Throughput } \\
\text { (Mbyte/Min) }\end{array}$ \\
\hline 1 & 50.0 & 1509.7 & 1509.7 & 377.4 & 187.5 \\
\hline 1 & 200.0 & 1313.3 & 750.0 & 407.5 & 203.4 \\
\hline 1 & 1000 & 1257.4 & 750.0 & 399.3 & 196.7 \\
\hline 381 & 85.0 & 1020.7 & 1275.0 & 510.3 & 212.5 \\
\hline 3386 & 455.1 & 989.1 & 1335.3 & 460.4 & 224.4 \\
\hline 22216 & 1000.0 & 672.1 & 1153.8 & 427.7 & 269.1 \\
\hline
\end{tabular}

The results showed that the average write time of the VTL tape in 1000M Ethernet is $99.14 \%$ of that in $100 \mathrm{M}$ Ethernet and the average read time in $1000 \mathrm{M}$ Ethernet is $95.68 \%$ of that in $100 \mathrm{M}$ Ethernet, which indicated that the different network environment has few influence for VBAS. However, the test results in local node indicate that network has large influence for VBAS. For instance, the average write time of the VTL tape in local host is $54.62 \%$ of that in $100 \mathrm{M}$ Ethernet and the average read time in local host is $61.64 \%$ of that in $100 \mathrm{M}$ Ethernet.

At the same time, the size of single file much affects the write performance of VBAS. For example, Table 1 and Table 2 show the write throughput are descending with the ascending number of file. Moreover, the average backup speed of the VTL 
tape in local node is 2.65 times of that of the physical tape and the average restore speed of the VTL tape in local node is 3.77 times of that of the physical tape, which indicate that the VTL tape in VBAS can enhance the backup speed greatly. So we can get a conclusion that our VBAS is fit for backup and restore applications, specially, for large size file.

\section{Conclusion and the Future Work}

It is important to note that simply replacing tape with low-cost disk will not provide the technological advantages. A disk-based backup solution can provide for smaller backup windows, and also provide somewhat faster recovery of distinct data files. But again, a disk-based solution does not provide off-site protection. VBAS significantly improves backup and restore by enabling all the data to remain online for faster, consistent restores. VBAS opens doors for new strategic applications by removing the cost and complexity of large quantities of traditional disk storage. The other traditional file system problems regarding performance and security are also no longer a concern. This is due to the fact that VBAS is typically proprietary and designed to act similar to a tape system with performance of disks. So, it is the primary benefits from archiving for end users to reduce in primary disk space and to improve performance (as expressed in faster response times).

\section{References}

1. Website, May, 2005, http://www.adic.com/

2. Paul Feresten. Comparing Host-Based D2D to VTLs for Backup and Restore - Part 2. Website, 2005. http://www.wwpi.com/index.php?option=com_content\&task=view\&id= $132 \&$ Itemid $=67$

3. T. E. Anderson, M. D. Dahlin, J. M. Neefe, D. A. Patterson, D. S. Roselli, and R. Y. Wang. Serverless network file systems. ACM Transactions on Computer Systems, 14(1):41-79, February 1996.

4. Chris Lueth, Network Appliance, Inc. October, 2004. NetApp Data Protection: Double Parity RAID for Enhanced Data Protection with RAID-DP. March 5, 2005, available from: http://www.netapp.com/tech_library/3298.html

5. J. Satran et al. Internet Small Computer Systems Interface (iSCSI). Available from: http://www.ietf.org/rfc/rfc3720.txt,April 2004.

6. Website, January 10, 2005, http://savannah.gnu.org/projects/tar/

7. Website, January 10, 2005, http://www.e-survey.net.au/taper/

8. Website, January 10, 2005, http://www.linux.org/apps/AppId_8816.html

9. Ashish A. Palekar etc. Design and Implementation of a Linux SCSI Target for Storage Area Networks. Proceedings of the 5th Annual Linux Showcase \& Conference, 2001.

10. Mu Fei, SHU Ji-wu, Li Bigang, ZHENG Wei-min. A Virtual Tape System Based on Storage Area Networks. H. Jin, Y Pan, N. Xiao and J. Sun (Eds.), GCC'2004 Workshop on Storage Grid and Technologies, LNCS 3252, pp.278-285, 2004.

11. Jussi Myllymaki,Miron Livny. Disk-tape joins: synchronizing disk and tape access. ACM SIGMETRICS Performance Evaluation Review, pp.279 - 290, 1995.

12. ANSI, SCSI Stream Commands-2 (SSC-2), revision 09, 9 July 2003, http://www.t10.org 